\title{
Depletion of potential $A 2 M$ risk haplotype for Alzheimer's disease in long-lived individuals
}

\author{
Friederike Flachsbart $^{1}$, Amke Caliebe ${ }^{2}$, Michael Nothnagel ${ }^{2}$, Rabea Kleindorp ${ }^{1}$, Susanna Nikolaus ${ }^{3,4}$, \\ Stefan Schreiber ${ }^{\star, 1,4}$ and Almut Nebel ${ }^{1}$
}

Risk alleles for age-related diseases are expected to decrease in frequency in the population strata of increasing age. Consistent with this hypothesis, earlier studies showed a depletion of the Alzheimer's disease risk factor $A P O E^{\star} \varepsilon 4$ in long-lived individuals (LLIs). To evaluate whether this observation also holds for a previously suggested Alzheimer's disease risk haplotype in the A2M gene, we analyzed this particular haplotype in 1042 German LLls (aged 95-100 years) and 1040 younger individuals (aged 60-75 years). Our results show a significant depletion of this haplotype in LLIs, thus confirming it as a mortality factor in the elderly. Consequently, our data support an involvement of the suggested A2M risk haplotype in the pathogenesis of Alzheimer's disease and adds new evidence to the risk-allele depletion hypothesis.

European Journal of Human Genetics (2010) 18, 59-61; doi:10.1038/ejhg.2009.136; published online 29 July 2009

Keywords: A2M; alpha2-macroglobulin; centenarians; longevity; Alzheimer's disease; age-related diseases

\section{INTRODUCTION}

The serum pan-protease inhibitor, alpha2-macroglobulin $(A 2 M)$, has been implicated as a plausible candidate for Alzheimer's disease (AD). The $A 2 M$ gene lies in an $\mathrm{AD}$ linkage region on chromosome $12 \mathrm{p} .{ }^{1,2}$ Two A2M polymorphisms, rs3832852 and rs669, have been widely tested for single-point association with $\mathrm{AD}$ in different populations, albeit with conflicting results. ${ }^{3,4}$ Interestingly, rs669 was also shown to be associated with argyrophilic grain disease, ${ }^{5}$ a late-onset form of dementia that is often reported with neurofibrillary lesions, which are also typical of AD. ${ }^{6}$ Recent meta-analyses of rs3832852 and rs669 for association with $\mathrm{AD}$ did not show any differences in allele frequencies between cases and controls in populations of European descent. Even after stratification for the $A P O E$ epsilon 4 allele $\left(A P O E^{\star} \varepsilon 4\right)$ as an established risk factor for $\mathrm{AD},{ }^{7}$ no differences were observed. ${ }^{4,8}$ However, haplotype analyses of the two $A 2 M$ polymorphisms in $A P O E^{\star} \varepsilon 4^{\text {negative }}$ patients by Verpillat et $a l^{9}$ supported the initially assumed association with AD.

Long-lived individuals (LLIs, nonagenarians and centenarians) have outlived the vast majority of their peers by many decades. Most of them have spent their life in good health and show a marked delay in the onset of or escape from age-related diseases, including AD. ${ }^{10,11}$ Longevity is considered a multi-factorial phenotype, and genetic factors have been estimated to account for about $25 \%$ of the variation in an individual's life expectancy. ${ }^{12}$ It has been postulated that LLI carry a reduced number of risk alleles for agerelated diseases compared with younger individuals. ${ }^{13}$ As carriers of such unfavorable variants are affected by higher mortality, they are expected to decrease in frequency in the population strata of increasing age. However, verification of this hypothesis is still ongoing. So far, the validity of this assumption has only been confirmed for the $A P O E^{\star} \varepsilon 4$ allele (risk factor for $\mathrm{AD}$ and for coronary heart disease), which is significantly depleted in LLI. ${ }^{12}$ To evaluate whether such a depletion is also observed for the previously described A2M-risk haplotype, ${ }^{9}$ we analyzed a large group of $>2000$ German centenarians/nonagenarians and younger individuals aged 60-75 years.

\section{MATERIALS AND METHODS}

\section{Participants}

In this study, we analyzed 2082 DNA samples drawn from German populationbased collections. The LLI sample comprised 1042 unrelated study participants of exceptional age (age range: 95-110 years, mean: 98.4 years), including 414 centenarians (mean age: 101.3 years). The LLI were required to be mentally fit to answer a health and family history questionnaire, thus excluding individuals affected by $\mathrm{AD}$ at the time of recruitment. The 1040 unrelated individuals of the younger comparison group (age range: 60-75 years, mean: 66.9 years) were drawn from a population-representative collection. They match the LLI in terms of ancestry, gender and geographical origin within the country, and genetic differences due to population stratification between the case-control samples are considered to be very low. ${ }^{14}$ The good matching is reflected in a genomic inflation factor $\lambda$ of $\leq 1.07$ for the whole LLI sample or the centenarian and nonagenarian subsets, respectively using PLINK V1.06; ${ }^{15}$ http://pngu.mgh.harvard.edu/purcell/plink/ (based on 290 randomly chosen genome-wide SNPs). A detailed description of the samples and the recruitment procedure is given elsewhere. ${ }^{16}$ All participants gave written informed consent before participation. The study was approved by the Ethics Committee of the University Hospital Schleswig-Holstein in Kiel.

\section{Genotyping}

We genotyped the two polymorphisms using a TaqMan SNP Genotyping Assay (rs3832852) and the SNPlex Genotyping System (rs669), both from Applied Biosystems, Foster City, CA, USA.

${ }^{1}$ Institute of Clinical Molecular Biology, Christian-Albrechts University and University Hospital Schleswig-Holstein, Kiel, Germany; ${ }^{2}$ Institute of Medical Informatics and Statistics, Christian-Albrechts University and University Hospital Schleswig-Holstein, Kiel, Germany; ${ }^{3}$ Popgen Biobank, Christian-Albrechts University and University Hospital SchleswigHolstein, Kiel, Germany; ${ }^{4}$ Department of General Internal Medicine, Christian-Albrechts University and University Hospital Schleswig-Holstein, Kiel, Germany

${ }^{*}$ Correspondence: Professor Dr S Schreiber, Institute of Clinical Molecular Biology, Christian-Albrechts University and University Hospital Schleswig-Holstein, Schittenhelmstraße 12, 24105 Kiel, Germany. Tel: +49 431597 2350; fax: +49 431597 1842. E-mail: s.schreiber@mucosa.de

Received 2 April 2009; revised 16 June 2009; accepted 24 June 2009; published online 29 July 2009 


\section{Statistical analyses}

An exact test in the genetics package of $\mathrm{R},{ }^{17}$ version 2.5.1, was applied to test for deviations from the Hardy-Weinberg equilibrium (HWE). Single-marker association analyses were conducted by logistic regression in R. Haplotype analyses for the complete sample and with regard to $A P O E^{\star} \varepsilon 4$ allele carriership by subgroup analyses were carried out with cocaphase (http://www.mrc-bsu. cam.ac.uk/personal/frank/software/unphased/). ${ }^{18} \mathrm{R}^{17}$ and the haplo.stats package $^{19}$ (http://www.R-project.org and http://cran.r-project.org/web/packages/ haplo.stats/) were used to test for haplotypic association under a multiplicative genetic model in our complete sample while adjusting for the number of $A P O E^{\star} \varepsilon 4$ alleles present. Sample size and power calculations were carried out with the statistics program BIAS. for Windows, version 8.03 (http:// www.bias-online.de/index.html)

\section{RESULTS}

Both SNPs showed no significant departure from HWE in cases and controls $\left(\mathrm{P}_{\mathrm{HWE}}>0.05\right)$. Single-marker comparisons did not show any significant association with or without adjustment for the $A P O E^{\star} \varepsilon 4$ risk factor, and no interaction with $A P O E$ was observed (data not shown). Haplotype analyses for rs3832852 and rs669 were first carried out for the complete sample without adjusting for the number of $A P O E^{\star} \varepsilon 4$ alleles. The previously reported rare $A 2 M^{\star} 2-G$ AD risk haplotype (haplotype definition see legend to Table 1$)^{9}$ was significantly less frequent in LLI compared with population-based younger individuals ( $P$ value $=0.002, \mathrm{OR}=0.09$, Table 1 , footnote a). The evidence for association was similar in the adjusted analysis (Table 1, footnote b), indicating a signal independent of the APOE status. When we additionally conducted a subgroup analysis with regard to $A P O E$ status $\left(A P O E^{\star} \varepsilon 4^{\text {negative }}\right.$ versus $A P O E^{\star} \varepsilon 4^{\text {positive }}$ individuals), the $A 2 M^{\star} 2-G$ haplotype was found to be significantly depleted in LLI in the $A P O E^{\star} \varepsilon 4^{\text {negative }}$ group. The comparison in the $A P O E^{\star} \varepsilon 4^{\text {positive }}$ individuals did not reach statistical significance, probably because of the small sample size and consequently low power. However, the frequency distribution was comparable in the two groups (Table 2), supporting the notion that in this study the $A P O E$ status does not seem to influence the observed association with AD. Furthermore, as the age of the study participants is of major concern for the analysis, we divided the complete LLI sample into a nonagenarian ( $n=628$, aged 95-99 years, mean age 96.5 years) and a centenarian subset ( $n=414$, mean age 101.3 years). As expected, the association was stronger in the centenarians than in nonagenarians (Table 3).

\section{DISCUSSSION}

The centenarians and nonagenarians have avoided or survived the most important age-related diseases that are responsible for the high mortality in the elderly. As the majority of them shows a favorable course of the ageing process they have been suggested to represent the so-called 'healthy-ageing' phenotype. ${ }^{11,20}$ In our study, the frequency of the rare potential $A 2 M^{\star} 2-\mathrm{G}$ risk haplotype for $\mathrm{AD}$ is significantly

\section{Table 1 Results of the $A 2 M$ haplotype analysis for long-lived and} younger individuals

\begin{tabular}{lccccc}
\hline & LLI & YI & $P$ value & OR & $P$ value \\
\hline Global significance & & & 0.007 & & 0.03 \\
A2M*1-A & $0.472(983)$ & $0.493(1026)$ & 0.27 & 1.00 & 0.23 \\
A2M*1-G & $0.354(738)$ & $0.331(689)$ & 0.21 & 1.12 & 0.15 \\
A2M*2-A & $0.173(361)$ & $0.166(344)$ & 0.77 & 1.10 & 0.89 \\
A2M*2-G & $0.00087(2)$ & $0.0099(21)$ & 0.002 & 0.09 & 0.01 \\
\hline
\end{tabular}

LLI, long-lived individuals; OR, odds ratio; YI, younger individuals.

a $P$ value and odds ratio for attaining old age obtained from haplotype analysis.

b $P$ value obtained from haplotype analysis after adjustment for $A P O E^{*} \varepsilon 4$ alleles.

Columns 2 and 3 present estimated A2M haplotype frequencies (absolute numbers are in parentheses).

The $A 2 M$ haplotype is shown in the variant order rs3832852 and rs669; rs3832852 has the two alleles, $A 2 M^{*} 1(+5 \mathrm{bp})$ and $A 2 M^{*} 2(-5 \mathrm{bp})$, and rs669 has the two alleles, $\mathrm{A}$ and $\mathrm{G}$

(haplotype designation according to Verpillat et al) ${ }^{9}$.

Table 2 Results of the APOE status-defined subgroup analysis of the $A 2 M$ haplotype (APOE* $84^{\text {negative }}$ versus $\mathrm{APOE}^{*} \varepsilon^{\text {positive }}$ individuals)

\begin{tabular}{|c|c|c|c|c|c|c|c|c|}
\hline & \multicolumn{4}{|c|}{ APOE* ${ }^{*} 4^{\text {neg }}$ individuals } & \multicolumn{4}{|c|}{ APOE ${ }^{*} 4^{\text {pos }}$ individuals } \\
\hline & $L L I$ & $Y I$ & $P$ value ${ }^{a}$ & $O R^{a}$ & LLI & $Y I$ & $P$ value ${ }^{b}$ & $O R^{\mathrm{b}}$ \\
\hline Global significance & & & 0.02 & & & & 0.32 & \\
\hline $\mathrm{A} 2 \mathrm{M} * 1-\mathrm{A}$ & $0.473(843)$ & $0.497(753)$ & 0.23 & 1.00 & $0.461(129)$ & $0.484(272)$ & 0.69 & 1.00 \\
\hline $\mathrm{A} 2 \mathrm{M} * 1-\mathrm{G}$ & $0.353(629)$ & $0.320(485)$ & 0.08 & 1.16 & $0.364(102)$ & $0.360(202)$ & 0.90 & 1.06 \\
\hline$A 2 M * 2-A$ & $0.173(308)$ & $0.174(263)$ & 0.75 & 1.05 & $0.175(49)$ & $0.143(80)$ & 0.36 & 1.29 \\
\hline$A 2 M * 2-G$ & $0.00098(2)$ & $0.0087(13)$ & 0.01 & 0.12 & $0.000(0)$ & $0.014(8)$ & 0.15 & $3.6 \times 10^{-9}$ \\
\hline
\end{tabular}

$A P O E^{*} \varepsilon 4^{\text {neg }}$, individuals without $A P O E^{*} \varepsilon 4$ allele carriership; $A P O E^{*} \varepsilon 4^{\text {pos }}$, individuals with $A P O E^{\star} \varepsilon 4$ allele carriership; LLI, long-lived individuals; OR, odds ratio; $Y$ I, younger individuals.

a $P$ value and odds ratio for attaining old age obtained from haplotype analysis for $A P O E^{*} \varepsilon 4^{\text {neg }}$ individuals.

${ }^{b} P$ value and odds ratio for attaining old age obtained from haplotype analysis for $A P O E^{*} \varepsilon 4^{\text {Pos }}$ individuals.

Columns 2, 3, 6 and 7 present estimated $A 2 M$ haplotype frequencies (absolute numbers are in parentheses).

Table 3 Results of the $\mathbf{A 2 M}$ haplotype analysis for the nonagenarian and centenarian subsets

\begin{tabular}{|c|c|c|c|c|c|c|c|}
\hline & Y (I 60-75 years) & Nonagenarians (95-99 years) & $P$ value ${ }^{\mathrm{a}}$ & $O R^{a}$ & Centenarians (100-110 years) & $P$ value ${ }^{\mathrm{b}}$ & $O R^{\mathrm{b}}$ \\
\hline Global significance & & & 0.085 & & & 0.017 & \\
\hline $\mathrm{A} 2 \mathrm{M} * 1-\mathrm{A}$ & $0.493(1026)$ & $0.475(597)$ & 0.44 & 1.00 & $0.466(386)$ & 0.27 & 1.00 \\
\hline$A 2 M * 1-G$ & 0.331 (689) & $0.358(449)$ & 0.19 & 1.12 & 0.349 (289) & 0.49 & 1.11 \\
\hline$A 2 M * 2-A$ & $0.166(344)$ & $0.166(208)$ & 0.75 & 1.04 & $0.185(153)$ & 0.34 & 1.18 \\
\hline$A 2 M * 2-G$ & $0.0099(21)$ & $0.0016(2)$ & 0.03 & 0.17 & $0.000(0)$ & 0.006 & $1.89 \times 10^{-10}$ \\
\hline
\end{tabular}

YI, younger individuals.

a $P$ value and odds ratio for attaining old age obtained from the haplotype analysis comparing the younger individuals with the nonagenarians

${ }^{b} P$ value and odds ratio for attaining old age obtained from the haplotype analysis comparing the younger individuals with the centenarians.

Columns 2, 3 and 6 present estimated $A 2 M$ haplotype frequencies (absolute numbers are in parentheses). 
lower in LLI compared with that of population-based younger individuals (Table 1). In the centenarian subset, the haplotype was completely absent (Table 3 ).

It is still not clear whether the associated risk haplotype is pathogenic and therefore decreased in LLI, or whether it is in linkage disequilibrium with another, as yet unknown, functional variant. Contrary to the findings of Verpillat et $a l,{ }^{9}$ in which an association between these haplotypes and $\mathrm{AD}$ was found only in $A P O E^{\star} \varepsilon 4^{\text {negative }}$ individuals, the $A P O E$ status does not seem to modify the observed association in our study, as evidence for the association was similar in the adjusted and the non-adjusted analyses. Furthermore, the distribution of haplotype frequencies was very similar in the complete sample and the $A P O E^{\star} \varepsilon 4$ strata (Tables 1 and 2).

The $A 2 M^{\star} 2-\mathrm{G}$ haplotype is rare in both LLI $(0.09 \%)$ and younger individuals $(0.99 \%)$. Therefore, a well-powered study of haplotype association requires a sample size at least as large as in this study. Here, we used a collection of altogether over 2000 individuals yielding a power of 0.70 (for comparison, for a power of 0.95, a sample size of 2000 individuals per group would be needed). For low haplotype frequencies it is particularly important to take the uncertainty of the haplotype estimation into account. We therefore applied two software programs that use different estimation and simulation procedures. Confidence in our findings is corroborated by the very similar results obtained from both programs.

The decrease in haplotype frequency that we observed in the population strata of increasing age characterizes the $A 2 M^{\star} 2-\mathrm{G}$ haplotype as a mortality risk factor in the elderly. Our findings are compatible with the results of Verpillat et al, ${ }^{9}$ who identified this haplotype as being significantly more frequent in $\mathrm{AD}$ individuals, suggesting this haplotype as a risk factor for $\mathrm{AD}$ and preventing those individuals from achieving extraordinarily old ages. Hence, our data support an involvement of the $A 2 \mathrm{M}$ risk haplotype in the pathogenesis of $\mathrm{AD}$ and adds new evidence to the risk-allele depletion hypothesis. ${ }^{13}$ To further strengthen this hypothesis, additional studies investigating the effect of other potential mortality factors are needed.

\section{CONFLICT OF INTEREST}

The authors declare no conflict of interest.

\section{ACKNOWLEDGEMENTS}

We thank all study participants for their cooperation. We acknowledge the laboratory personnel of the Institute of Clinical Molecular Biology for excellent technical assistance and the staff of the Popgen Biobank for expert help with the sample recruitment. This study was supported by the DFG excellence cluster
'Inflammation at Interfaces' and the German Ministry of Education and Research (BMBF) through the National Genome Research Network (NGFN-2).

1 Blacker D, Wilcox MA, Laird NM et al: Alpha-2 macroglobulin is genetically associated with Alzheimer disease. Nat Genet 1998; 19: 357-360.

2 Liao A, Nitsch RM, Greenberg SM et al: Genetic association of an alpha2-macroglobulin (Val1000lle) polymorphism and Alzheimer's disease. Hum Mol Genet 1998; 7: 1953-1956.

3 Saunders AJ, Bertram L, Mullin K et al: Genetic association of Alzheimer's disease with multiple polymorphisms in alpha-2-macroglobulin. Hum Mol Genet 2003; 12: 2765-2776.

4 Panza F, Colacicco AM, D'Introno A et al: Candidate genes for late-onset Alzheimer's disease: focus on chromosome 12. Mech Ageing Dev 2006; 127: 36-47.

5 Ghebremedhin E, Schultz C, Thal DR, Del Tredici K, Rueb U, Braak H: Genetic association of argyrophilic grain disease with polymorphisms in alpha-2 macroglobulin and low-density lipoprotein receptor-related protein genes. Neuropathol Appl Neurobiol 2002; 28: 308-313.

6 Probst A, Tolnay M: Argyrophilic grain disease (AgD), a frequent and largely underestimated cause of dementia in old patients. Rev Neurol (Paris) 2002; 158: $155-165$.

7 Corder EH, Saunders AM, Strittmatter WJ et al: Gene dose of apolipoprotein E type 4 allele and the risk of Alzheimer's disease in late onset families. Science 1993; 261: 921-923.

8 Koster MN, Dermaut B, Cruts M et al: The alpha2-macroglobulin gene in AD: a population-based study and meta-analysis. Neurology 2000; 55: 678-684.

9 Verpillat P, Bouley S, Hannequin D et al: Alpha2-macroglobulin gene and Alzheimer's disease: confirmation of association by haplotypes analyses. Ann Neurol 2000; 48: 400-402.

10 Hitt R, Young-Xu Y, Silver M, Perls T: Centenarians: the older you get, the healthier you have been. Lancet 1999; 354: 652.

11 Evert J, Lawler E, Bogan H, Perls T: Morbidity profiles of centenarians: survivors, delayers, and escapers. J Gerontol A Biol Sci Med Sci 2003; 58: 232-237.

12 Christensen K, Johnson TE, Vaupel JW: The quest for genetic determinants of human longevity: challenges and insights. Nat Rev Genet 2006; 7: 436-448.

13 Perls T, Kunkel LM, Puca AA: The genetics of exceptional human longevity. J Am Geriatr Soc 2002; 50: 359-368.

14 Flachsbart F, Caliebe A, Kleindorp R et al: Association of FOXO3A variation with human longevity confirmed in German centenarians. Proc Natl Acad Sci USA 2009; 106: 2700-2705.

15 Purcell S, Neale B, Todd-Brown K et al: PLINK: a tool set for whole-genome association and population-based linkage analyses. Am J Hum Genet 2007; 81: 559-575.

16 Nebel A, Croucher PJ, Stiegeler R, Nikolaus S, Krawczak M, Schreiber S: No association between microsomal triglyceride transfer protein (MTP) haplotype and longevity in humans. Proc Natl Acad Sci USA 2005; 102: 7906-7909.

$17 \mathrm{R}$ Development Core Team: A Language and Environment for Statistical Computing. R Foundation for Statistical Computing: Vienna, Austria, ISBN 3-900051-07-0 2008, URL http://www.R-project.org.

18 Dudbridge F: Pedigree disequilibrium tests for multilocus haplotypes. Genet Epidemiol 2003; 25: 115-121.

19 Schaid DJ, Rowland CM, Tines DE, Jacobson RM, Poland GA: Score tests for association between traits and haplotypes when linkage phase is ambiguous. $A m$ Hum Genet 2002; 70: 425-434.

20 Franceschi $\mathrm{C}$, Bonafé M: Centenarians as a model for healthy aging. Biochem Soc Trans 2003; 31: 457-461. 